Original article

\title{
EFFECTS OF ANTIGLUCOCORTICOID PRETREATMENT ON TESTES IN CHRONICALLY STRESSED ADULT RATS - A HISTOMORPHOMETRIC STUDY
}

\author{
M. KARAMI KHEIRABAD ${ }^{1}$, B. NAMAVAR JAHROMI ${ }^{2}$, F. RAHMANIFAR ${ }^{3}$, \\ A. TAMADON ${ }^{4}$, M. OWJFARD $^{4}$, N. TANIDEH ${ }^{4,5} \&$ O. KOOHI-HOSSEINABADI ${ }^{6}$ \\ ${ }^{1}$ Department of Basic Sciences, Azad University, Gachsaran branch, Gachsaran, Iran; ${ }^{2}$ In- \\ fertility Research Center, Department of OB-GYN, School of Medicine, Shiraz University \\ of Medical Sciences, Shiraz, Iran; ${ }^{3}$ Department of Basic Sciences, School of Veterinary \\ Medicine, Shiraz University, Shiraz, Iran; ${ }^{4}$ Transgenic Technology Research Center, Shiraz \\ University of Medical Sciences, Shiraz, Iran; ${ }^{5}$ Department of Pharmacology, School of Me- \\ dicine, Shiraz University of Medical Sciences, Shiraz, Iran; ${ }^{6}$ Laboratory Animal Center, \\ Shiraz University of Medical Sciences, Shiraz, Iran
}

\section{Summary}

Karami Kheirabad, M., B. Namavar Jahromi, F. Rahmanifar, A. Tamadon, M. Owjfard, N. Tanideh \& O. Koohi-Hosseinabadi, 2016. Effects of antiglucocorticoid pretreatment on testes in chronically stressed adult rats - a histomorphometric study. Bulg. J. Vet. Med., 2016, 19, No 4, 281-289.

Male fertility and reproduction can be affected by stress. The aim of the present study was histomorphometric evaluation of the role of glucocorticoids during chronic restraint stress on spermatogenesis. Twenty four male adult Sprague-Dawley rats were allocated randomly to four equal groups; stress group, mifepristone (RU486)-treated group, stress/RU486 and control groups. In stress group, the rats were restrained $1 \mathrm{~h}$ /day for 12 days. In RU486 group, the rats were injected RU486 at a dose of 2.5 $\mathrm{mg} / \mathrm{kg}$ for 12 days. In stress/RU486 group, the rats were injected RU486 $1 \mathrm{~h}$ before the stress process for 12 days. The testes of the all groups were removed and on 10 circular transverse sections of tubules stained with hematoxylin-eosin, histomorphometric parameters including cellular (germinal epithelium) diameter and area of the seminiferous tubules, total diameter and cross sectional area, number of seminiferous tubules per unit area, and numerical density of the tubules of the seminiferous tubules were measured. Restraint stress reduced lumen diameter, thickness of germinal epithelium and numerical density of seminiferous tubules $(\mathrm{P}<0.05)$. This reduction was reversed by subcutaneous injection of the anti-glucocorticoid, RU486 prior to stress session $(\mathrm{P}<0.05)$. In conclusion, chronic stress acts on testicular tissue via glucocorticoid receptors to suppress spermatogenesis in male rats.

Key words: chronic stress, glucocorticoid receptor, histomorphometry, rats, spermatogenesis 
Effects of antiglucocorticoid pretreatment on testes in chronically stressed adult rats ....

\section{INTRODUCTION}

Stressful events can disrupt endocrine signalling and reproduction (Chand \& Lovejoy, 2011). The secretion of glucocorticoids is a typical response to stress. Previous studies in rats attempted to correlate stress-induced glucocorticoid secretion with alterations in the structure of the testis and sub-fertility (Yazawa et al., 2000; Mingoti et al., 2003; Swami \& Jeganathan Ramanathan, 2007). The mediators of the effects of stress on the hypothalamic-pituitary-gonadal (HPG) axis include glucocorticoids, catecholamines, arginine-vasopressin, corticotropin-releasing factor (CRF), and endogenous opioids. Many of the negative effects of stress on fertility may be attributed to elevated production of stress hormones, especially glucocorticoids, within the circulation and testes. These mediators exert disruptive effects on spermatogenic cells. Hypothalamus neurons express abundant glucocorticoid receptors (Rosenfeld et al., 1988) and dexamethasone as a synthetic glucocorticoid suppresses transcription in immortalised gonadotropin-releasing hormone $(\mathrm{GnRH})$ secreting hypothalamic cell lines (Chandran et al., 1994). In addition, immunohistochemical studies showed a large density of glucocorticoid receptors at the interstitial and basal zone of the testis in rats (Stalker et al., 1989; Biagini et al., 1995).

In a chronically stressed rat model, plasma corticosterone concentration was increased, whereas plasma testosterone was decreased (Yazawa et al., 2000). This association has led to the general argument that glucocorticoids play a profound role in mediating the inhibitory effects of stress on reproduction. In addition, glucocorticoids increase the inhibitory actions of gonadotropin-inhibitory hormone $(\mathrm{GnIH})$, a novel negative regulator of re- productive axis, on GnRH secretion (Kirby et al., 2009). Even after sexual development, psychophysical stressors exert control over reproduction, causing abnormal estrous cycling and decreased sexual hormone production. Because of the complexity of stress response, how the HPG axis senses external stressors remains to be understood.

Mifepristone (RU486) is a derivative of the progestin norethindrone, and acts as a progesterone receptor antagonist or glucocorticoid receptor antagonist (Cadepond et al., 1997; Munhoz et al., 2006). An advantage of glucocorticoid receptor antagonists is that prolonged antagonism of glucocorticoid receptor may actually result in glucocorticoid receptor up-regulation in hypothalamus and limbic structures. Therefore, it enhances the glucocorticoid receptor negative feedback control of the hypothalamic-pituitary-adrenal (HPA) axis (Wulsin et al., 2010). Moreover, the use of RU486 in individuals with elevated glucocorticoids as a result of chronic stress may be particularly advantageous as preliminary blockade of glucocorticoid receptor can serve as a barrier against the deleterious effects of excess glucocorticoid production (Wulsin et al., 2010). RU486 normalised changes in adult neurogenesis induced after chronic stress in rats (Oomen et al., 2007). RU486 rapidly reversed a chronic corticosterone-induced reduction of adult neurogenesis in rats (Mayer et al., 2006).

Chronic stress was found to suppress expression of $\mathrm{GnIH}$, related to reproductive system (Karami Kheirabad et al., 2015). Systemic administration of RU486 partially reversed the restraint-induced decrease in $\mathrm{GnIH}$ expression, confirming that glucocorticoids and its receptor are 
involved in steroidogenic suppression (Karami Kheirabad et al., 2015).

The aim of the present study was to determine the effects of chronic exposure to restraint stress on testes of rats and evaluate the use of RU486 as an antiglucocorticoid agent at testicular level. Using histomorphometric analysis of histological sections at the light microscope level to follow changes in volume, surface area and other structures in three dimensions to be calculated from the two dimensional images of light microscopy.

\section{MATERIALS AND METHODS}

\section{Animals}

Twenty four male adult Sprague-Dawley rats weighing 220-240 g were housed in the Laboratory Animal Center, Shiraz University of Medical Sciences, Iran under controlled temperature $\left(23 \pm 1^{\circ} \mathrm{C}\right)$, 12:12-h light/dark cycle, and $55 \pm 5 \%$ relative humidity. They were given standard pellet and water ad libitum during the experimental period. The experimental investigation was approved by Ethics committee of Shiraz University of Medical Sciences.

The rats were randomly divided into four equal groups $(n=6)$ : stress, RU486, stress/RU486, and control groups. Restraint stress was performed daily for 12 days. Briefly, rats were handled before experiments. After that, animals were individually restrained for $1 \mathrm{~h}$ through wrapping of their upper and lower limbs by plastic cylinders $(20.5 \times 8 \times 6 \mathrm{~cm})$ with holes for ventilation and their extended tails. The cylinders were just large enough to allow rats of the size used to turn around easily. In RU486 group, the rats were subcutaneously injected RU486 (2.5 $\mathrm{mg} / \mathrm{kg}, 20 \mu \mathrm{L} / \mathrm{rat}$; ab120356, Abcam Ltd, Cambridge, UK) for 12 days. In
stress/RU486 group, the rats were subcutaneously injected with the same dose of RU486, 60 min before the stress process for 12 days. The control group was allowed to freely move in the cages and did not receive any stress or drug. All of the experiments were conducted between 8:00 and 12:00 AM.

\section{Histomorphometric analysis}

Twenty four hours after the last session of stress, the rats were weighted and anaesthetised by inhalation of ether and euthanised by cervical dislocation. For histological evaluation, the right testes of all groups were dissected out and weighed using a digital weighing scale (precision $0.001 \mathrm{~g}$ ). The testicular tissues were fixed in fresh $10 \%$ buffered formalin solution in room temperature. After that each testis was implanted in paraffin. Ethanol and xylene were used for dehydration step. Samples were embedded in paraffin wax and sectioned at thicknesses of $5 \mu \mathrm{m}$. Sections were deparaffinised at $60{ }^{\circ} \mathrm{C}$ and dehydrated in graded concentrations of xylene and ethanol rehydrated in room temperature and stained with haematoxylin and eosin stain. Finally, sections were viewed and photographed on light microscope (CX21, Olympus, Japan) equipped with an adjusted digital camera (AM423U Eyepiece Camera, Dino-Eye, Taiwan) and histomorphometric indices were measured using Dinocapture 2.0 software (DinoEye, Taiwan). Counts used to determine the numerical densities and the volume of seminiferous tubules were made with a $\times 40$ objective lens and the same eyepiece grid.

Total, lumen and cellular diameters $(\mu \mathrm{m})$, lumen, cellular and cross sectional area $\left(\times 10^{4} \mu \mathrm{m}^{2}\right)$, number of tubules (per $\left.5 \times 5 \mathrm{~mm}^{2}\right)$ and numerical density were determined randomly in 10 circular trans- 
Effects of antiglucocorticoid pretreatment on testes in chronically stressed adult rats ....

verse sections of different regions of testes (Panahi et al., 2015a).

The mean seminiferous tubule diameter (D) was measured in 10 seminiferous tubules per animal by taking the average of two diameters, D1 and D2 at right angles. Cross-sectional area (Ac) of the seminiferous tubules was determined using the equation $\mathrm{Ac}=\pi(\mathrm{D} / 2)^{2}$, where $\pi$ is equivalent to 3.142 and $\mathrm{D}$ is the mean diameter of seminiferous tubules (Panahi et al., 2015b). The number of seminiferous tubules per unit area (NA) was determined using the unbiased counting frame proposed by Gundersen et al., (1988). Numerical density (Nv) of seminiferous tubules was the number of seminiferous tubules per unit volume and it was using the modified Floderus equation (Panahi et al., 2015a):

$$
\mathrm{Nv}=\mathrm{NA} /(\mathrm{D}+\mathrm{T})
$$

where NA is the number of seminiferous tubules per unit area, D is the mean diameter of the seminiferous tubule and $\mathrm{T}$ is the average thickness of the section $(\mu \mathrm{m})$.

\section{Statistical analysis}

The data of histomorphometric indices of seminiferous tubules were subjected to Kolmogorov-Smirnov test of normality and analysed by one-way ANOVA and LSD post hoc test (SPSS for Windows, version 20, SPSS Inc, Chicago, Illinois). The P-value of less than 0.05 was considered to be statistically significant. Group means and their standard error were reported in the text and graphs (GraphPad Prism version 5.01 for Windows, GraphPad software Inc., San Diego, CA, USA).

\section{RESULTS}

There was no significant difference in body weights (g, Fig. 1A) and volume of testis $\left(\mathrm{cm}^{3}\right.$, Fig. 1B) between rats in all groups $(\mathrm{P}>0.05)$. Testes in all groups presented tubules with thin basement membrane and tunica propria; as well as normal germinal epithelium showing orderly progression from spermatogonia to spermatocytes with groups of spermatids and mature spermatozoa (Fig. 2A). Sertoli cells (Epitheliocytus sustentans) were compressed between the germinal cells and were not easily seen. The interstitium contained normal numbers of Leydig cells (Endocrinocytus interstitialis) (Fig. 2B).

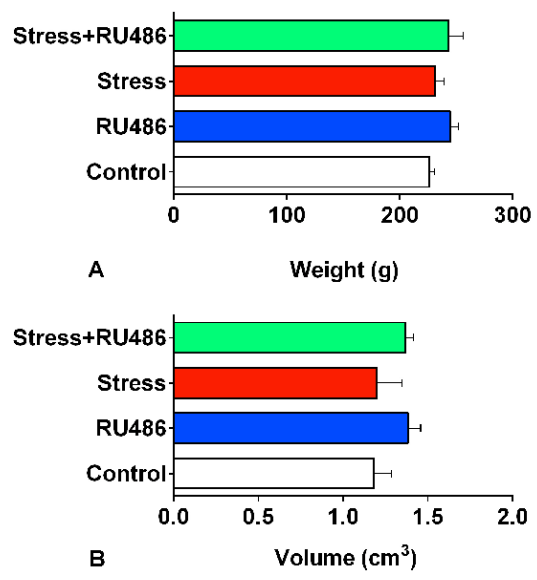

Fig. 1. Mean \pm standard error weight $(A)$ and testis volume (B) in the different groups to evaluate the effects of chronic stress on testes of adult male rats.

In stereological analysis, lumen diameter of the seminiferous tubules in the stress group was higher than both control and stress/RU486 groups $(\mathrm{P}<0.05$; Fig. $3 \mathrm{~A})$. Furthermore, luminal area of the seminiferous tubules in RU486 and stress groups was higher vs the stress/RU486 group ( $\mathrm{P}<0.05$; Fig. 4A). Moreover, cellular diameter and cellular area in stress/RU486 group were bigger than those of other groups $(\mathrm{P}<0.05$; Figs. $3 \mathrm{~B}$ and $4 \mathrm{~B})$. In addition, cellular diameter and 

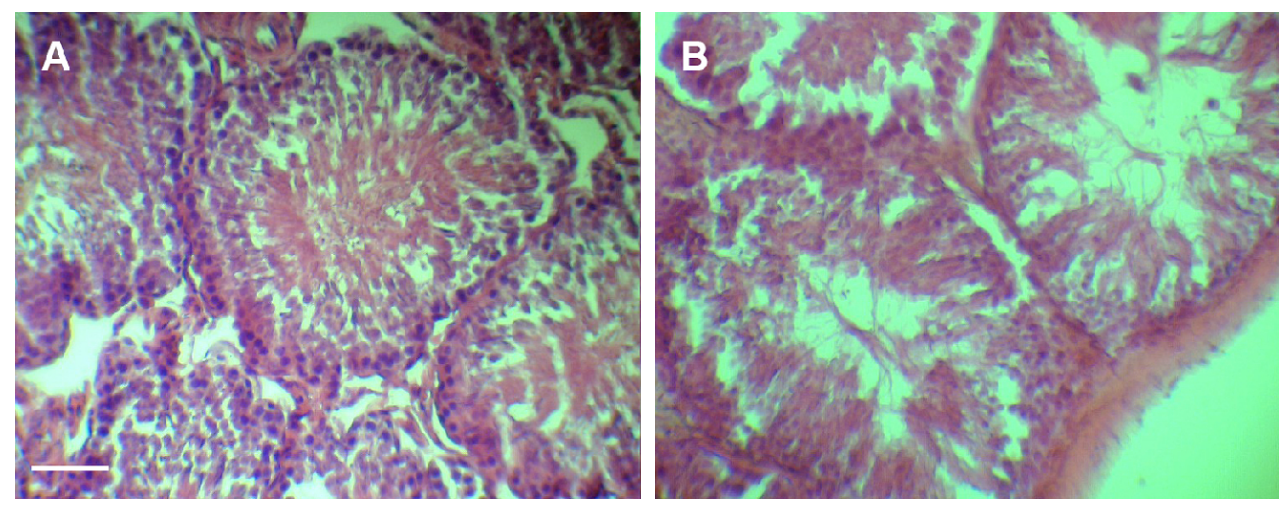

Fig. 2. Seminiferous tubules in control rats (A) and rats with chronic stress (B). Seminiferous tubules with thin basement membrane and tunica propria; as well as normal germinal epithelium showing orderly progression from spermatogonia to spermatocytes. $\mathrm{H} \& \mathrm{E}$, bar $=50 \mu \mathrm{m}$.
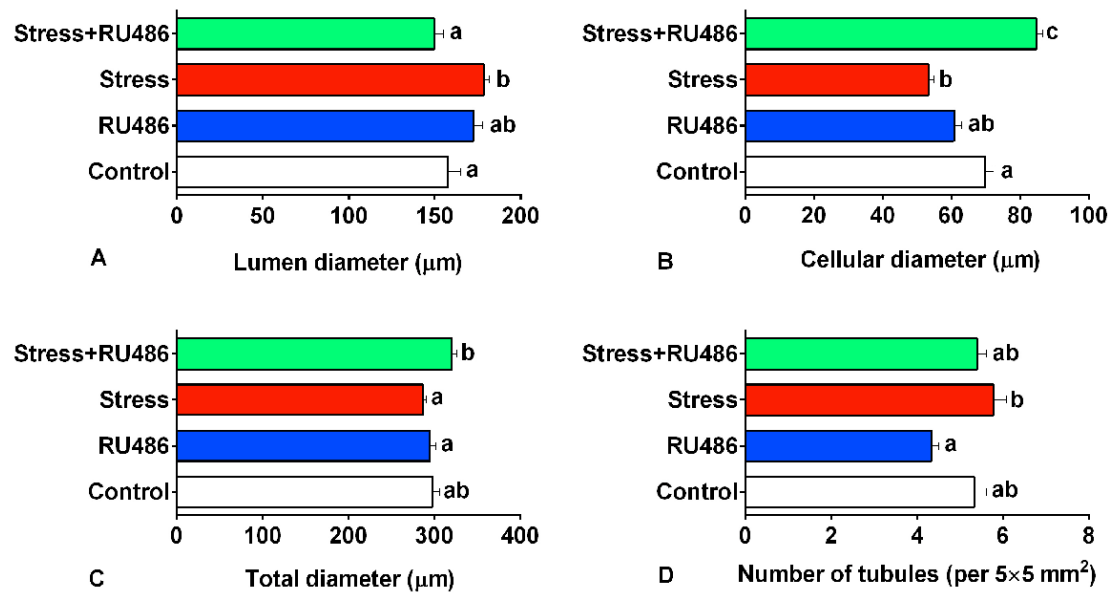

Fig. 3. Mean \pm standard error of histomorphometric indices of seminiferous tubules in different groups to evaluate the effects of chronic stress on testes of adult male rats. A. Lumen diameter $(\mu \mathrm{m})$; B. Cellular diameter $(\mu \mathrm{m})$; C. Total diameters $(\mu \mathrm{m})$; D. Luminal area $\left(\times 10^{4} \mu \mathrm{m}^{2}\right)$. ${ }^{\mathrm{a}, \mathrm{b}, \mathrm{c}}$ Different letters show significant differences between groups.

cellular area in stress group were inferior to the control group $(\mathrm{P}<0.05)$. Altogether, total diameter of the seminiferous tubules in RU486 and stress groups was lower than stress/ RU486 group $(\mathrm{P}<0.05$; Fig. $3 \mathrm{C})$. However, cross sectional area of the seminiferous tubules in stress/RU486 group was larger compared to the stress group $(\mathrm{P}<0.05$; Fig. 4C). Moreover, the number of seminiferous tubules per unit area of testis in stress group was higher than that in the RU486 group $(\mathrm{P}<0.05$; Fig. 3D) while numerical density of the seminiferous tubules in RU486 and stress groups was lower than the stress/RU486 group ( $\mathrm{P}<0.05$; Fig. 4D). 

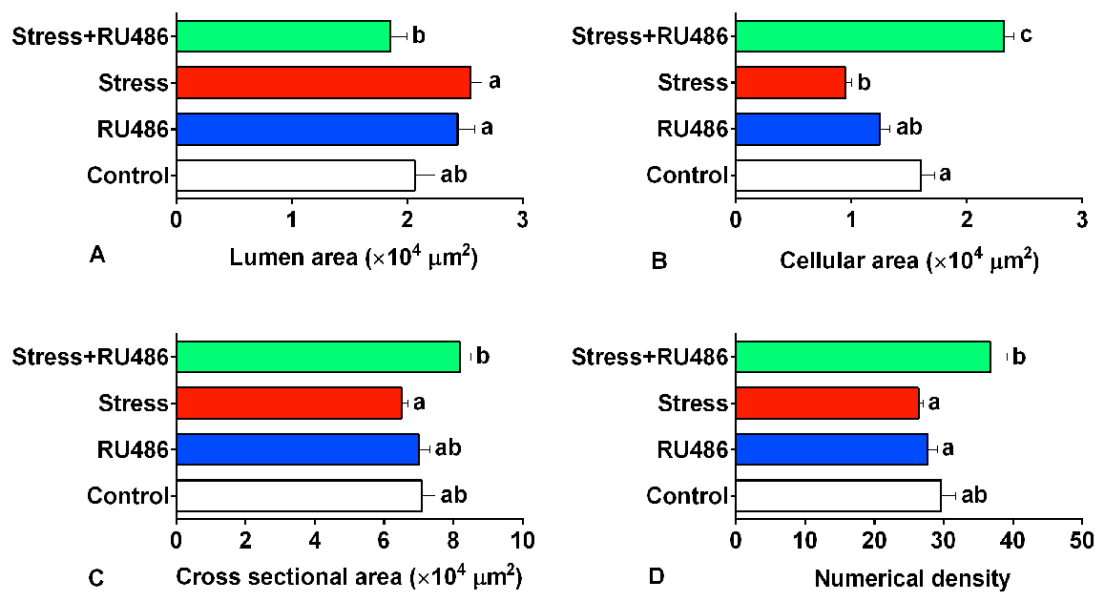

Fig. 4. Mean \pm standard error of histomorphometric indices of seminiferous tubules in different groups to evaluate the effects of chronic stress on testes of adult male rats. A. Cellular area $\left(\times 10^{4} \mu \mathrm{m}^{2}\right)$; B. Cross sectional area of the tubule $\left(\times 10^{4} \mu \mathrm{m}^{2}\right)$; C. Number of seminiferous tubules per unit area of testis (per $5 \times 5 \mathrm{~mm}^{2}$ ) and D. numerical density of the seminiferous tubules. ${ }^{\text {a,b,c }}$ Different letters show significant differences between groups.

\section{DISCUSSION}

Twelve consecutive days of restraint stress caused morphometric alterations, i.e. lumen diameter and cellular area were decreased that might be the result of an impairment in the division and conversion of the spermatogonia to the spermatozoa. Consistent with our findings, RetanaMárquez et al. (2014) observed that epithelial surface area decreased in testis of male rats stressed for 20 days. In addition, Nirupama et al. (2013) reported that following restraint stress, the seminiferous tubules of rats were shrunken and contained less spermatozoa. Moreover, after chronic restraint stress in rat, decrease in both the production of maturing spermatids and concentration of spermatozoa in the cauda epididymidis was observed (Almeida et al., 1998). Following the RU486 treatment of chronic stress in the present study, thickness of germinal epithelium increased. The chronic restraint stress had a negative action on the epithelium of the seminiferous tubules; it provoked a moderate tubular hypocellularity. There are evidences that stress-induced increases in corticosterone are directly responsible for Leydig cell apoptosis (Bernier et al., 1984; Dong et al., 2004). Localisation of glucocorticoid receptor in the testis and Leydig cells suggests that different stressors directly affect steroidogenesis in the testis (Schultz et al., 1993). Therefore, the effects of chronic stress are most pronounced on the seminiferous epithelium and restraint stress in the rats led to damage to spermatogenesis.

Following chronic restraint stress, body weights and testis volume did not change. Consistent with our results, Almeida et al. (1998) reported that no change was observed in the testicular weight following chronic restraint stress. In contrast, 
testicular weights decreased on days 20 and 50 in stressed male rats (Retana-Márquez et al., 2014). Present observations of impaired spermatogenesis with chronic stress suggested that spermatogenesis in rats might have resulted from the alteration in the seminiferous tubules milieu due to testosterone deficiency. These findings suggest that tubular cells can be a target of stress. The stress-induced suppression of spermatogenesis was blocked by RU486, an antagonist of type II glucocorticoid receptors, implying that elevation in plasma corticosterone mediates the stressinduced reduction of spermatogenesis via action on this receptor. Furthermore, recent work has demonstrated an up-regulation of hypothalamic RFamide-related peptide-3 (RFRP-3) mRNA on chronically restrained rats (Karami Kheirabad et $a l ., 2015)$. There is evidence that RFRP-3 neurons express functional glucocorticoid receptors by which stress can influence reproductive function (Kirby et al., 2009). In addition, adrenalectomy blocks the increase in RFRP-3 expression (Kirby et al., 2009). Moreover, stress decreases the sensitivity of gonads in male rats to gonadotropins. This may be caused by reducing LH receptor (Orr \& Mann, 1990; 1992). It is not well understood whether the excessive glucocorticoid is responsible for decrease in spermatogenesis activity directly, or affects interstitial cells in the testis and thereby contributes to the stressinduced suppression of spermatogenesis. It should be noted that RU486 might act centrally to improve spermatogenesis.

\section{CONCLUSIONS}

The exposure to chronic restraint stress impairs spermatogenesis in rats via glucocorticoid receptors and seminiferous tubules can be a major target of stress. An- tiglucocorticoid pretreatment by mifepristone (RU486) reduced the effect of chronic stress on male rat subfertility.

\section{ACKNOWLEDGMENTS}

This study was supported by Infertility Research Center, Shiraz University of Medical Sciences, Shiraz, Iran. The authors would like to thank the staff of Transgenic Technology Research Center and Also Laboratory Animal Center, Shiraz University of Medical Sciences, for their assistance in this study.

\section{REFERENCES}

Almeida, S. A., S. O. Petenusci, J. A. Anselmo-Franci, A. A. M. Rosa-e-Silva \& T. L. Lamano-Carvalho, 1998. Decreased spermatogenic and androgenic testicular functions in adult rats submitted to immobilization-induced stress from prepuberty. Brazilian Journal of Medical and Biological Research, 31, 1443-1448.

Bernier, M., W. Gibb, R. Collu \& J. Ducharme, 1984. Effect of glucocorticoids on testosterone production by porcine Leydig cells in primary culture. Canadian Journal of Physiology and Pharmacology, 62, 1166-1169.

Biagini, G., E. M. Pich, A. Frasoldati, L. F. Agnati \& P. Marrama, 1995. Changes in glucocorticoid receptor immunoreactivity after adrenalectomy and corticosterone treatment in the rat testis. Journal of Endocrinological Investigation, 18, 384-390.

Cadepond, F., A. Ulmann \& E. E. Baulieu, 1997. RU486 (mifepristone): mechanisms of action and clinical uses. Annual Review of Medicine, 48, 129-156.

Chand, D. \& D. A. Lovejoy, 2011. Stress and reproduction: controversies and challenges. General and Comparative Endocrinology, 171, 253-257.

Chandran, U., B. Attardi, R. Friedman, K. Dong, J. L. Roberts \& D. B. DeFranco, 1994. Glucocorticoid receptor-mediated 
Effects of antiglucocorticoid pretreatment on testes in chronically stressed adult rats ....

repression of gonadotropin-releasing hormone promoter activity in GT1 hypothalamic cell lines. Endocrinology, 134, 1467-1474.

Dong, Q., A. Salva, C. M. Sottas, E. Niu, M. Holmes \& M. P. Hardy, 2004. Rapid glucocorticoid mediation of suppressed testosterone biosynthesis in male mice subjected to immobilization stress. Journal of Andrology, 25, 973-981.

Gundersen, H., T. F. Bendtsen, L. Korbo, N. Marcussen, A. Møller, K. Nielsen, J. Nyengaard, B. Pakkenberg, F. Sørensen \& A. Vesterby, 1988. Some new, simple and efficient stereological methods and their use in pathological research and diagnosis. APMIS, 96, 379-394.

Karami Kheirabad, M., S. Ahmadloo, B. Namavar Jahromi, A. Tamadon, A. Ramezani, M. Owjfard, F. Sabet Sarvestani \& O. Koohi-Hosseinabadi, 2015. RF-amide related peptide- 3 and Kiss 1 gene expressions in male rat hypothalamus during chronic stress. Veterinarski Arhiv (in press)

Kirby, E. D., A. C. Geraghty, T. Ubuka, G. E. Bentley \& D. Kaufer, 2009. Stress increases putative gonadotropin inhibitory hormone and decreases luteinizing hormone in male rats. Proceedings of the $\mathrm{Na}$ tional Academy of Sciences of the USA, 106, 11324-11329.

Mayer, J. L., L. Klumpers, S. Maslam, E. R. De Kloet, M. Joels, \& P. J. Lucassen, 2006. Brief treatment with the glucocorticoid receptor antagonist mifepristone normalises the corticosterone-induced reduction of adult hippocampal neurogenesis. Journal of Neuroendocrinology, 18, 629-631.

Mingoti, G. Z., R. Pereira \& C. Monteiro, 2003. Fertility of male adult rats submitted to forced swimming stress. Brazilian Journal of Medical and Biological Research, 36, 677-682.

Munhoz, C. D., L. B. Lepsch, E. M. Kawamoto, M. B. Malta, L. de Sá Lima, M. C. W. Avellar, R. M. Sapolsky \& C. Scavone, 2006. Chronic unpredictable stress exacerbates lipopolysaccharide-induced activa- tion of nuclear factor- $\kappa \mathrm{B}$ in the frontal cortex and hippocampus via glucocorticoid secretion. The Journal of Neuroscience, 26, 3813-3820.

Nirupama, M., M. Devaki, R. Nirupama \& H. N. Yajurvedi, 2013. Chronic intermittent stress-induced alterations in the spermatogenesis and antioxidant status of the testis are irreversible in albino rat. Journal of Physiology and Biochemistry, 69, 59-68.

Oomen, C. A., J. L. Mayer, E. R. De Kloet, M. Joëls, \& P. J. Lucassen, 2007. Brief treatment with the glucocorticoid receptor antagonist mifepristone normalizes the reduction in neurogenesis after chronic stress. European Journal of Neuroscience, 26, 3395-3401.

Orr, T. E. \& D. R. Mann, 1990. Effects of restraint stress on plasma $\mathrm{LH}$ and testosterone concentrations, Leydig cell $\mathrm{LH} / \mathrm{hCG}$ receptors, and in vitro testicular steroidogenesis in adult rats. Hormones and Behavior, 24, 324-341.

Orr, T. E. \& D. R. Mann, 1992. Role of glucocorticoids in the stress-induced suppression of testicular steroidogenesis in adult male rats. Hormones and Behavior, 26, 350-363.

Panahi, M., N. Karimaghai, F. Rahmanifar, A. Tamadon, A. Vahdati, D. Mehrabani, O. Koohi-Hosseinabadi \& M. Sepehrimanesh, 2015a. Stereological evaluation of testes in busulfan-induced infertility of hamster. Comparative Clinical Pathology, 24, 1051-1056.

Panahi, M., S. Keshavarz, F. Rahmanifar, A. Tamadon, D. Mehrabani, N. Karimaghai, M. Sepehrimanesh \& H. Aqababa, 2015. Busulfan induced azoospermia: stereological evaluation of testes in rat. Veterinary Research Forum (in press).

Retana-Márquez, S., R. M. Vigueras-Villaseñor, L. Juárez-Rojas, A. Aragón-Martínez, \& G. Reyes Torres, 2014. Sexual behavior attenuates the effects of chronic stress in body weight, testes, sexual accessory glands, and plasma testosterone in 
male rats. Hormones and Behavior, 66, 766-778.

Rosenfeld, P., J. Van Eekelen, S. Levine \& E. De Kloet, 1988. Ontogeny of the type 2 glucocorticoid receptor in discrete rat brain regions: An immunocytochemical study. Developmental Brain Research, 42, 119-127.

Schultz, R., J. Isola, M. Parvinen, J. Honkaniemi, A.-C. Wikström, J.-Å. Gustafsson \& M. Pelto-Huikko, 1993. Localization of the glucocorticoid receptor in testis and accessory sexual organs of male rat. $\mathrm{Mo}$ lecular and Cellular Endocrinology, 95, 115-120.

Stalker, A., L. Hermo \& T. Antakly, 1989. Covalent affinity labeling, radioautography, and immunocytochemistry localize the glucocorticoid receptor in rat testicular Leydig cells. American Journal of Anatomy, 186, 369-377.

Swami, C. G. \& C. Jeganathan Ramanathan, 2007. Noise exposure effect on testicular histology, morphology and on male steroidogenic hormone. The Malaysian Journal of Medical Sciences, 14, 28.

Wulsin, A. C., J. P. Herman, \& Ma. B. Solomon, 2010. Mifepristone decreases depression-like behavior and modulates neuroendocrine and central hypothalamic-pituitary-adrenocortical axis responsiveness to stress. Psychoneuroendocrinology, 35, 1100-1112.
Yazawa, H., I. Sasagawa \& T. Nakada, 2000. Apoptosis of testicular germ cells induced by exogenous glucocorticoid in rats. $\mathrm{Hu}$ man Reproduction, 15, 1917-1920.

Paper received 08.06.2015; accepted for publication 15.06.2015

\section{Correspondence:}

Bahia Namavar Jahromi Infertility Research Center,

Shiraz University of Medical Sciences, Ghadir Mother \& Child Hospital,

Quran Gate, Shiraz, Iran,

Tel/fax: +98-71-32279715,

e-mail: namavarb@sums.ac.ir

\section{Amin Tamadon}

Transgenic Technology Research Center, Shiraz University of Medical Sciences, Neshat ave., near Sina \& Sadra Halls, Shiraz, Iran, Postal code: 71348-74478, Tel/fax: +98-71-32341025,

e-mail: amintamaddon@yahoo.com 